\title{
O uso da aromaterapia na prática clínica e interprofissional
}

\author{
The use of aromatherapy in clinical and interprofessional practice \\ El uso de la aromaterapia en la práctica clínica e interprofesional
}

Débora Luana Ribeiro Pessoa

ORCID: https://orcid.org/0000-0002-9817-5647

Universidade Federal do Maranhão, Brasil

E-mail: debora.luana@ufma.br

Bruno Oliveira Santos

ORCID: https://orcid.org/0000-0002-2726-4685

Universidade Federal do Maranhão, Brasil

E-mail: brunooliveira_96@hotmail.com

Carla Bianca Rodrigues Abreu

ORCID: https://orcid.org/0000-0002-7345-5028

Universidade Federal do Maranhão, Brasil E-mail: bia.rodrigues.9698@gmail.com

Karen Fernanda Cruz Mendes

ORCID: https://orcid.org/0000-0001-5997-5513

Universidade Federal do Maranhão, Brasil E-mail: karenfernandajk@hotmail.com

Maria da Conceição Coelho da Hora

ORCID: https://orcid.org/0000-0002-0136-3685

Universidade Federal do Maranhão, Brasil

E-mail: maria.cch@ discente.ufma.br

Matheus Costa Cantanhede

ORCID: https://orcid.org/0000-0001-6388-0058 Universidade Federal do Maranhão, Brasil

E-mail: cantanhede.matheus@discente.ufma.br

Renata Ricciardi de Salles

ORCID: https://orcid.org/0000-0002-6272-5344 Universidade Federal do Maranhão, Brasil

E-mail: renata.ricciardi@discente.ufma.br

Sara Fiterman Lima

ORCID: https://orcid.org/0000-0003-0015-3413 Universidade Federal do Maranhão, Brasil

E-mail: sara.fiterman@ufma.br

Mayara Soares Cunha Carvalho

ORCID: https://orcid.org/0000-0003-2167-5675

Universidade Federal do Maranhão, Brasil E-mail: mayara.sc@ufma.br

Carlos Eduardo Neves Amorim

ORCID: https://orcid.org/0000-0001-6183-2503

Universidade Federal do Maranhão, Brasil

E-mail: amorim.carlos@ufma.br

\begin{abstract}
Resumo
A aromaterapia faz uso de óleos essenciais através de aplicação tópica, inalatória ou olfatória, objetivando a prevenção, a cura e a diminuição de sintomas, sendo uma das práticas complementares mais antigas do mundo, sendo reconhecida e empregada em muitos países, tanto no tratamento de problemas de saúde como na promoção do bemestar e da qualidade de vida. Este artigo tem como objetivo identificar as funções da aromaterapia nos cuidados com a saúde traçando um panorama de como está configurada, na literatura nacional e internacional, no que diz respeito a utilização da aromaterapia como ferramenta para a redução da ansiedade e promoção de bem-estar psicológico e a contribuição no trabalho interprofissional. Trata-se de uma revisão de literatura, onde a partir da busca de artigos nas bases Medline e Scielo, publicados no período de 2009 a 2018 e selecionados utilizando os descritores "ansiedade" e "aromaterapia", foram selecionados 18 artigos para o estudo. Deste modo acredita-se que este estudo contribuiu no âmbito da prática profissional, da formação acadêmica e na área científica, pois através do mesmo pôde se identificar eficácia na utilização de algumas práticas emergentes na atualidade, balizando a necessidade de repensar implementar modelos tanto de ensino como, de extensão e pesquisa, além dos atendimentos.
\end{abstract}

Palavras-chave: Óleos essenciais; Aromaterapia; Interprofissionalidade. 


\begin{abstract}
Aromatherapy makes use of essential oils through topical, inhalation or olfactory application, aiming at the prevention, cure and reduction of symptoms, being one of the oldest complementary practices in the world, being recognized and used in many countries, both in the treatment of health problems as well as promoting well-being and quality of life. This article aims to identify the functions of aromatherapy in health care by drawing an overview of how it is configured, in national and international literature, with regard to the use of aromatherapy as a tool for reducing anxiety and promoting psychological well-being. and the contribution to interprofessional work. It is a literature review, where from the search for articles in the Medline and Scielo databases, published between 2009 and 2018 and selected using the descriptors "anxiety" and "aromatherapy", 18 articles were selected for the study. In this way, it is believed that this study contributed in the scope of professional practice, academic training and in the scientific area, as through it, it was possible to identify the effectiveness in the use of some emerging practices nowadays, highlighting the need to rethink the implementation of both teaching models such as extension and research, in addition to assistance.
\end{abstract}

Keywords: Essential oils; Aromatherapy; Interprofessionality.

\title{
Resumen
}

La aromaterapia hace uso de aceites esenciales mediante aplicación tópica, inhalatoria u olfativa, con el objetivo de prevenir, curar y reducir los síntomas, siendo una de las prácticas complementarias más antiguas del mundo, siendo reconocida y utilizada en muchos países, tanto en el tratamiento de la salud. problemas, así como promover el bienestar y la calidad de vida. Este artículo tiene como objetivo identificar las funciones de la aromaterapia en el cuidado de la salud trazando un panorama de cómo se configura, en la literatura nacional e internacional, en cuanto al uso de la aromaterapia como herramienta para reducir la ansiedad y promover el bienestar psicológico. contribución al trabajo interprofesional. Se trata de una revisión de la literatura, donde a partir de la búsqueda de artículos en las bases de datos Medline y Scielo, publicados entre 2009 y 2018 y seleccionados mediante los descriptores "ansiedad" y "aromaterapia", se seleccionaron 18 artículos para el estudio. De esta manera, se cree que este estudio contribuyó en el ámbito de la práctica profesional, la formación académica y en el área científica, ya que a través de él se pudo identificar la efectividad en el uso de algunas prácticas emergentes en la actualidad, destacando la necesidad de repensar la implementación tanto de modelos de enseñanza como extensión e investigación, además de asistencial.

Palabras clave: Aceites esenciales; Aromaterapia; Interprofesionalidad.

\section{Introdução}

Em maio de 2006, através da portaria ํ9 971, o Ministério da Saúde aprovou a Política Nacional de Práticas Integrativas e Complementares no Sistema Único de Saúde que inicialmente contemplava apenas 5 práticas: Homeopatia, Fitoterapia, Acupuntura, Medicina Antrofosófica e Termalismo Social. Em 2017, a portaria N 849, de 27 de março de 2017 oficializou e legitimou mais 14 novas práticas e, em 2018, mais $10 \mathrm{com}$ a portaria $\mathrm{n}^{\circ} 702$, dentre estas a Aromaterapia (Brasil, 2018).

Segundo a portaria $\mathrm{N}^{\circ}$ 702, de 21 de março de 2018 do Ministério da Saúde, a aromaterapia é prática terapêutica secular que consiste no uso intencional de concentrados voláteis extraídos de vegetais - os óleos essenciais (OE) - a fim de promover ou melhorar a saúde, o bem-estar e a higiene. Na década de 30, a França e a Inglaterra passaram a adotar e pesquisar o uso terapêutico dos óleos essenciais, sendo considerada prática integrante da aromatologia. No Brasil, a aromaterapia é reconhecida como uma prática integrativa e complementar com amplo uso individual e/ou coletivo, podendo ser associada a outras práticas como talassoterapia e naturopatia, e considerada uma possibilidade de intervenção que potencializa os resultados do tratamento adotado (Macedo, 2018).

A aromaterapia faz uso de óleos essenciais através de aplicação tópica, inalatória ou olfatória, objetivando a prevenção, a cura e a diminuição de sintomas. Segundo Gnatta et al. (2011), quando atuam através do olfato, as moléculas dos óleos são absorvidas pelos nervos olfativos, os quais têm uma ligação direta com o sistema nervoso central e levam o estímulo ao sistema límbico, sendo este responsável pelos sentimentos, memórias, impulsos e emoções. Quando a atuação é via cutânea, as moléculas são absorvidas e caem na circulação sanguínea, sendo transportadas para os tecidos e órgãos do corpo.

Os óleos essenciais são compostos orgânicos de origem vegetal que apresentam várias funções químicas, como alcoóis, aldeídos, entre outras, havendo sempre a prevalência de uma ou duas delas. Esses óleos são obtidos a partir da 
destilação ou prensagem de flores, folhas, sementes, frutos e raízes (Gnatta, Zotelli, et al., 2011). Nos vegetais, os óleos auxiliam na proteção contra agentes hostis e doenças, e contribuem para a fertilização e polinização. Na prática clínica os óleos são utilizados como antissépticos, antibactericidas, anti-inflamatórios, antiespamódicos, expectorantes, entre outras funções (Andrei \& Del Comune, 2005).

A aromaterapia é uma das práticas complementares mais antigas do mundo, sendo reconhecida e empregada em muitos países, tanto no tratamento de problemas de saúde como na promoção do bem-estar e da qualidade de vida. Apesar disso, a literatura científica ainda carece de estudos conclusivos comprovando a sua eficácia, para servir de embasamento teórico e subsidiar a prática assistencial.

A integração de trabalhos pela equipe, em oposição ao agrupamento de agentes, é especialmente importante para refletir sobre o trabalho interprofissional com as Práticas Integrativas e Complementares (PIC) na Atenção em Saúde (Barros et al., 2018), e esta integração para que todos os membros atuem em conjunto é uma característica do trabalho interprofissional.

Desta forma, entende-se que este tipo de pesquisa contribui para a construção de conhecimentos acerca do uso de técnicas não farmacológicas e norteia a tomada de decisão do profissional, possibilitando um cuidado holístico em saúde, alicerçado na cientificidade.

Neste contexto, o presente estudo tem como objetivo identificar as funções da aromaterapia nos cuidados com a saúde traçando um panorama de como está configurada, na literatura nacional e internacional, no que diz respeito a utilização da aromaterapia como ferramenta para a redução da ansiedade e promoção de bem-estar psicológico, e como o trabalho interprofissional pode contribuir nesta prática.

\section{Metodologia}

Para contemplar os objetivos propostos nesta pesquisa, foi escolhida como método a revisão integrativa de literatura, uma vez que este método tem como principal característica a contextualização do pesquisador em relação a temática em análise, abrindo margem para o fomento de conhecimento e a identificação de lacunas da evidência e do conhecimento na prática.

Domingo \& Braga (2013) citam que o rigor metodológico limita o viés no levantamento das publicações, na análise crítica e na síntese dos resultados; e se constitui no cumprimento de cada uma das seis etapas, a saber: (1) Identificação do tema e seleção da hipótese de pesquisa; (2) Estabelecimento de critérios para inclusão e exclusão de estudos; (3) Definição das informações a serem extraídas dos estudos selecionados/Categorização dos estudos; (4) Avaliação dos estudos selecionados; (5) Interpretação dos resultados e (6) Síntese do conhecimento.

Para a primeira etapa desta revisão integrativa elaborou-se uma pergunta norteadora, a saber: "Como se estabelece, na literatura nacional e internacional, a utilização da aromaterapia como ferramenta para a redução da ansiedade e promoção de bem-estar psicológico?".

Diante disso, foram eleitas Medline (Medical Literature Analysis and online) e Scielo (Scientific Eletronic Library Online) como base de dados para pesquisa. Ainda, foram estabelecidos como critérios de inclusão: pesquisas publicadas de 2009 a 2018; nos idiomas inglês, português, espanhol e francês; selecionadas a partir das palavras-chaves "ansiedade" e "aromaterapia" e seus correlatos em inglês. A amostragem dos artigos inclui revisões sistemáticas e integrativas, e estudos com delineamento experimental e quase-experimental.

Como critérios de exclusão, citam-se: artigos publicados em datas inferiores a 2008, artigos que se repetiam nas bases de dados, artigos cujos objetivos não contemplavam esta pesquisa, artigos publicados em idiomas diferentes dos adotados, artigos de acesso restrito e documentos como editoriais, resumos, dissertações e teses.

No total, foram encontrados 692 artigos potenciais para análise, dos quais 17 artigos pertenciam a base de dados 
Scielo, 675 na Medline. Considerando os critérios de inclusão e exclusão supracitados, a seleção se constituiu de 18 artigos analisados por meio de instrumento validado nacionalmente.

Optou-se por organizar a apresentação dos resultados partindo de uma descrição geral dos artigos selecionados, seguido de tabelas que ilustram descritivamente os principais achados de cada artigo selecionado e por fim, uma discussão apontando as lacunas de conhecimento e as perspectivas para pesquisas e ações em aromaterapia.

\section{Resultados e Discussão}

A Tabela 1 apresenta um resumo dos estudos selecionados que avaliam os objetivos, metodologia e principais resultados e conclusões sobre aromaterapia, que posteriormente serão discutidos.

Tabela 1. Síntese dos artigos selecionados sobre aromaterapia.

\begin{tabular}{|c|c|c|c|c|c|}
\hline Autores & $\begin{array}{l}\text { Local } \\
\text { do } \\
\text { Estudo }\end{array}$ & Objetivo & Metodologia & Resultado & Conclusão \\
\hline $\begin{array}{l}\text { Gnatta, Jr. } \\
\text { et al } \\
(2010)\end{array}$ & Brasil & $\begin{array}{l}\text { Investigar o uso da } \\
\text { aromaterapia no } \\
\text { alívio da ansiedade }\end{array}$ & $\begin{array}{l}\text { Estudo de campo, } \\
\text { experimental, com } \\
\text { abordagem quantitativa, } \\
\text { realizado com os } \\
\text { discentes do primeiro } \\
\text { ano de Graduação da } \\
\text { Escola de Enfermagem } \\
\text { da Universidade de São } \\
\text { Paulo }\end{array}$ & $\begin{array}{l}\begin{array}{l}\text { observou-se que não } \\
\text { houve } \\
\text { significantes }\end{array} \\
\text { estatisticamente }\end{array}$ & $\begin{array}{l}\text { Verificou-se que o OE de } \\
\text { Lavanda diminuiu o estado de } \\
\text { ansiedade ao final de } 60 \text { dias de } \\
\text { uso, porém de forma não } \\
\text { significativa estatisticamente }\end{array}$ \\
\hline $\begin{array}{l}\text { Montibele } \\
\text { r, J. et al } \\
(2018)\end{array}$ & Brasil & $\begin{array}{l}\text { Efetividade da } \\
\text { massagem rom } \\
\text { aromaterapia no } \\
\text { estresse da equipe } \\
\text { de enfermagem do } \\
\text { centro cirúrgico: } \\
\text { estudo-piloto }\end{array}$ & $\begin{array}{l}\text { Estudo-piloto com } \\
\text { delineamento de ensaio } \\
\text { clínico controlado e } \\
\text { randomizado, } \\
\text { desenvolvido em um } \\
\text { centro cirúrgico de um } \\
\text { hospital de ensino no } \\
\text { interior de São } \\
\text { Paulo/Brasil }\end{array}$ & $\begin{array}{l}\text { A análise } \\
\text { frequência cástica da } \\
\text { demonstrou redução } \\
\text { significativa na totalidade } \\
\text { das sessões de } \\
\text { aromaterapia, } \\
\text { confirmando a efetividade } \\
\text { da intervenção na } \\
\text { diminuição do estresse } \\
\text { dos profissionais da } \\
\text { equipe de saúde. }\end{array}$ & $\begin{array}{l}\text { A aromaterapia associada à } \\
\text { massagem demonstrou } \\
\text { efetividade na redução de } \\
\text { parâmetros biofisiológicos dos } \\
\text { profissionais da equipe de } \\
\text { enfermagem de centro } \\
\text { cirúrgico, redução essa } \\
\text { evidenciada pela diminuição } \\
\text { estatisticamente significativa da } \\
\text { frequência cardíaca e pressão } \\
\text { arterial }\end{array}$ \\
\hline $\begin{array}{l}\text { Gnatta, } \\
\text { J.R. et al } \\
(2010)\end{array}$ & Brasil & $\begin{array}{lr}\text { Investigar o uso da } \\
\text { aromaterapia } & \text { na } \\
\text { melhora } & \text { da } \\
\text { autoestima } & \end{array}$ & 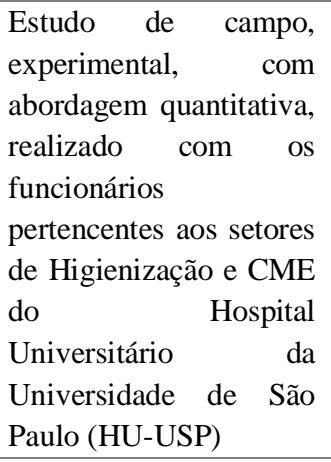 & $\begin{array}{l}\text { Não houve diferença } \\
\text { significativa entre os } \\
\text { grupos na melhora da } \\
\text { autoestima }\end{array}$ & $\begin{array}{l}\text { De acordo com os objetivos } \\
\text { propostos, verificou-se que os } \\
\text { óleos essenciais de rosa e } \\
\text { ylang-ylang não alteraram de } \\
\text { forma significativa a percepção } \\
\text { da autoestima. }\end{array}$ \\
\hline $\begin{array}{l}\text { Domingos } \\
\text { T. S.; } \\
\text { Braga, E. } \\
\text { M (2014) }\end{array}$ & Brasil & $\begin{array}{lr}\text { Significado } & \mathrm{da} \\
\text { massagem } & \mathrm{com} \\
\text { aromaterapia } & \mathrm{em} \\
\text { saúde mental } & \end{array}$ & $\begin{array}{l}\text { Pesquisa de campo, } \\
\text { descritiva, exploratória, } \\
\text { de abordagem } \\
\text { qualitativa, utilizando } \\
\text { como instrumento de } \\
\text { coleta de dados } \\
\text { entrevista. }\end{array}$ & $\begin{array}{l}\text { Os sujeitos da pesquisa } \\
\text { atribuíram à aromaterapia } \\
\text { a função de favorecer o } \\
\text { bem-estar psicológico e } \\
\text { físico durante o período } \\
\text { de internação, auxiliando- } \\
\text { os na adaptação ao } \\
\text { ambiente e diminuindo }\end{array}$ & $\begin{array}{l}\text { A intervenção com } \\
\text { aromaterapia na internação } \\
\text { psiquiátrica em hospitais gerais } \\
\text { trouxe melhorias em diversas } \\
\text { esferas, como a diminuição dos } \\
\text { sintomas ansiosos e no } \\
\text { enfretamento do transtorno } \\
\text { mental }\end{array}$ \\
\hline
\end{tabular}




\begin{tabular}{rllrlll}
\hline Domingos & Brasil & Massagem & com & Ensaio clínico não & Diminuição significativa \\
T. S.; & aromaterapia: & & controlado realizado em & da frequência cardíaca e \\
Braga, E. & efetividade sobre a & uma unidade de & respiratória após cada \\
M(2015) & ansiedade & de & internação psiquiátrica & sessão de intervenção \\
& usuários & com & em hospital geral no & \\
& transtornos & de & interior do estado de \\
& personalidade & em & São Paulo & \\
& internação & &
\end{tabular}

A intervenção de massagem com aromaterapia durante a internação psiquiátrica para pacientes diagnosticados com TP demonstrou ser efetiva para a diminuição da ansiedade

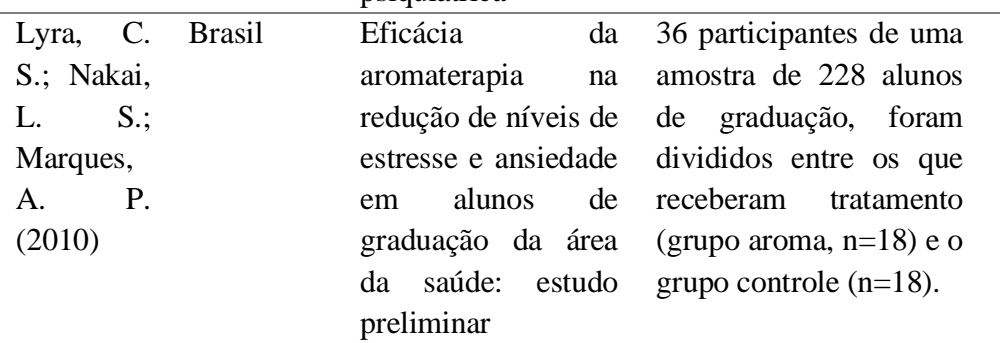

Gnatta, Brasil Verificar como o Discentes do $1^{\circ}$ ano de

J.R.; uso do óleo Graduação da Escola de essencial (OE) de Lavanda e Gerânio altera a percepção do estado de tensão e ansiedade

Enfermagem foram randomizados em 3 grupos: dois que receberam $\mathrm{OE}$ (em um gel de base polimérica)
Dornellas,

E.V;

Silva,

M.J.P

(2011) e o outro placebo (que recebeu essência de Rosa).

$\begin{array}{ll}\text { Farahani, Irã } & \begin{array}{l}\text { Determinar o efeito } \\ \text { da aromaterapia nas }\end{array} \\ \text { (2019) } & \\ & \begin{array}{l}\text { complicações do } \\ \text { câncer. }\end{array}\end{array}$

Revisão sistemática de

43 ensaios clínicos randomizados, realizada a partir de pesquisas em bases de dados internacionais nacionais.

\section{Gnatta, Brasil}

J.R. et al

(2014)

\begin{tabular}{ll}
\hline Ali, B. et & Arábia \\
al (2015) & Saudita
\end{tabular}

Verificar se o uso do óleo essencial de ylang ylang altera a percepção ansiedade e da autoestima.
34 profissionais da equipe de enfermagem foram randomizados em três grupos: um recebeu o óleo essencial de ylang ylang via cutânea, o segundo o recebeu via inalatória e o terceiro (placebo) recebeu essência de ylang ylang via cutânea.

\begin{tabular}{|c|c|c|c|}
\hline $\begin{array}{l}\text { Explorar } \\
\text { informações } \\
\text { disponíveis } \\
\text { literatura } \\
\text { questões } \\
\text { terapêuticas, } \\
\text { médicas, } \\
\text { cosméticas, } \\
\text { psicológicas, } \\
\text { olfativas, } \\
\text { aromaterapia }\end{array}$ & $\begin{array}{r}\text { na } \\
\text { sobre }\end{array}$ & $\begin{array}{l}\text { Revisão sistemática a } \\
\text { partir de bancos de } \\
\text { dados eletrônicos, } \\
\text { e pesquisa em } \\
\text { bibliotecas. }\end{array}$ & $\begin{array}{l}\text { Numerosos estudos foram } \\
\text { realizados para estudar os } \\
\text { efeitos dessa terapia no } \\
\text { cérebro humano e em suas } \\
\text { emoções. Seu papel no } \\
\text { humor, atenção e estresse } \\
\text { mental em indivíduos } \\
\text { saudáveis foi um tópico } \\
\text { de discussões entre a } \\
\text { comunidade científica } \\
\text { recentemente. }\end{array}$ \\
\hline
\end{tabular}

O grupo $\begin{array}{r}\text { aroma } \\ \text { redução }\end{array}$
$\begin{aligned} & \text { apresentou } \\ & \text { significativa dos níveis de }\end{aligned}$
estresse e ansiedade,
enquanto no grupo
controle houve uma
diminuição, apenas no
nível de ansiedade
$\mathrm{O}$ grupo que utilizou Lavanda teve uma redução de $-11,80$ na média dos escores, porém não significativa estatisticamente.
Houve maior eficácia do óleo de Lavanda na diminuição da ansiedade, porém não foi estatisticamente expressiva.
A aromaterapia mostrou-se eficaz na redução de estresse e ansiedade em estudantes da área da saúde.
A aromaterapia melhora as várias complicações físicas e psicológicas, embora poucos estudos tenham mostrado que a aromaterapia não teve efeito nas reações cutâneas.

Houve diferença significante para os três grupos na variável autoestima.

\section{A aromaterapia pode ser usada como um tratamento suplementar adequado para melhorar as complicações, embora sejam necessários mais estudos para determinar o protocolo e a dosagem padrão. \\ Houve alterações significativas apenas na percepção da ansiedade intragrupo para os três grupos.}


massagem, questões

de segurança e

diferentes plantas

usadas na

aromaterapia.

Paganini, Uso da Analisar as fichas

T.; Flores aromatera dos óleos essenciais

e Silva, Y. pia no utilizados no

(2014) combate combate da

ao estresse patologia do

estresse e verificar

seu uso, ações e

efeitos

da

aromaterapia para $\mathrm{o}$ organismo.

$\begin{array}{ll}\text { Gayeski, } & \text { Métodos não } \\ \text { M. E. e } & \text { farmacológicos para } \\ \text { Bruggema } & \text { alívio da dor no } \\ \text { nn, O. M. } & \text { trabalho de parto: } \\ \text { (2010) } & \text { uma revisão } \\ & \text { sistemática }\end{array}$

Através da busca dos Foram relacionados 27 materiais científicos por meio de livros, dissertações e artigos científicos plataforma Scielo

na óleos capazes proporcionar melhora significativa contra o estresse, diminuindo os efeitos ocasionados no corpo humano. por um profissional de qualidade, assim também como os óleos utilizados. Para que possa ser aplicada com mais segurança e assim aumentar a sua popularidade e segurança.

Revisão sistemática

através de pesquisa nas bases de dados CINAHL, MEDLINE, LILACS, SciELO, SCOPUS e Isi Web of Science.
Clínico Randomizado avaliou a aromaterapia durante o trabalho de parto em relação aos resultados maternos $\mathrm{e}$ neonatais. Não houve diferença nos resultados maternos referentes à cesárea, parto a vácuo, parto espontâneo vaginal e duração da primeira fase do trabalho de parto. A redução da dor foi mais percebida pelas nulíparas do Grupo Experimental antes e após a administração da aromaterapia, o que não ocorreu com as multíparas. A dor, a ansiedade e o medo foram menores para $86 \%$ das mulheres que receberam a intervenção. Em relação aos resultados neonatais, houve uma redução de admissões de bebês do Grupo Experimental.
Para cada método é necessário definir em que fase do período de dilatação, latente ou ativa, ele deve ser implementado. Para a aromaterapia, demonstrou ser um método que também reduz a ansiedade, além do medo, que pode influenciar positivamente nos resultados neonatais.

$\begin{array}{lrlllr}\text { Han X; } & \text { Estados } & \text { Observar os efeitos } & \text { Cinquenta } & \text { e } & \text { sete } \\ \text { Gibson J; } & \text { Unidos } & \text { da inalação do óleo } & \text { participantes elegíveis } \\ \text { Eggett } & & \text { essencial de } & (50 \text { mulheres, faixa } \\ \text { DL; } & & \text { bergamota na saúde } & \text { etária: 23-70 anos) } \\ \text { Parker TL } & & \text { mental e bem-estar. } & \text { foram incluídos para } \\ (2017) & & & \text { análise. }\end{array}$

Quinze minutos de
exposição ao óleo essencial de bergamota melhoraram sentimentos positivos dos participantes em comparação com o grupo controle $(17 \%$ maior)

O estudo fornece evidências preliminares da eficácia e segurança da inalação de óleo essencial de bergamota no bem-estar mental em saúde mental tratamento, sugerindo que a aromaterapia com óleo essencial de bergamota pode ser um eficaz tratamento adjunto para melhorar a saúde mental e o bem-estar dos indivíduos.

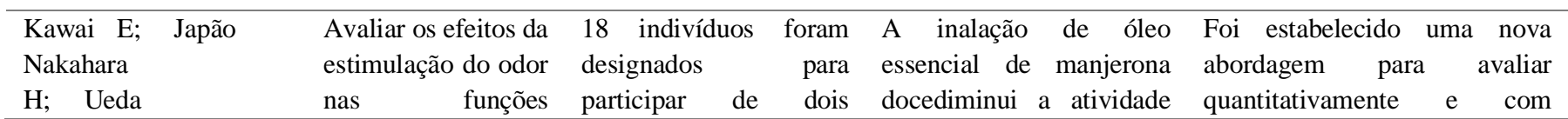




\section{SY;}

Manabe

$\mathrm{K}$;

Miyamoto

$\mathrm{T}(2017)$ cardiorrespiratórias protocolos em: um em

dinâmicas.

(1)

repouso e outro em decúbito dorsal. Os sujeitos respiravam o ar ambiente através de uma máscara facial. Para examinar os efeitos, cada indivíduo foi testado enquanto inalava ar contendo óleo essencial de manjerona doce e enquanto inalava o ar sem óleo essencial. do nervo simpático e pode induzir a progressão dependente do tempo dos efeitos depressores e bradicárdicos.

\begin{tabular}{|c|c|c|c|c|}
\hline \multicolumn{2}{|l|}{ Johnson } & Uso & de & Após uma pré-pesquisa, \\
\hline $\mathrm{K}$; West & Estados & aromaterapia & para & Óleo \\
\hline T; Diana & Unidos & promover & um & Essencial de Lavanda \\
\hline S; Todd J; & & ambiente & & foi difundido 24 horas \\
\hline Haynes B; & & terapêutico & para & por dia, durante 30 dias, \\
\hline Bernhardt & & enfermeiros & & em uma área de \\
\hline J; Johnson & & & & enfermagem designada, \\
\hline R (2017) & & & & $\begin{array}{l}\text { na qual nem todos os } \\
\text { enfermeiros deveriam } \\
\text { entrar em cada unidade. }\end{array}$ \\
\hline
\end{tabular}

Uma média pré-pesquisa de 2,97 ( $d p=0,99)$, que foi significativamente maior que a média póspesquisa $\quad 2,70$ $(d p=0,92) \quad$ com significância, $t(69)=2$, $36, p=0,021$, sugerindo uma diferença na frequência com que a equipe se sentiu estressada no trabalho em uma semana típica

$\begin{array}{lllll}\text { Turten } & \text { Turquia } & \text { Efeitos } & \text { da } & \text { Os pacientes e seus } \\ \text { Kaymaz } & \text { aromaterapia } & \text { na } & \text { cuidadores de dois } \\ \text { T; } & \text { agitação e na carga } & \text { hospitais da Turquia } \\ \text { Ozdemir } & \text { relacionada } & \text { ao } & \text { foram selecionados e } \\ \text { L (2016) } & \text { cuidador } & \text { em } & \text { divididos em um grupo } \\ & \text { pacientes com } & \text { de intervenção }(\mathrm{n}=14) \\ & \text { demência moderada } & \text { e um grupo de controle } \\ & \text { a grave: um estudo } & (14) . \\ & \text { piloto. } & n=14)\end{array}$

Lee YR; Coréia do Examinar a eficácia $\mathrm{O}$ grupo experimental Shin HS Sul da aromaterapia $(\mathrm{n}=30)$ recebeu (2015) com óleo essencial de gengibre na náusea inalação de óleo essencial de gengibre. $\mathrm{O}$ grupo controle e vômito em placebo $(\mathrm{n}=30)$ pacientes submetidos recebeu inalação salina cirurgia abdominal.

Agitação, sintomas neuropsiquiátricos angústia do cuidador reduziram significativamente, e a aromaterapia impediu o aumento da carga do cuidador.

Os escores de náusea e vômito foram significativamente menores no grupo experimental com inalação de óleo essencial de gengibre do que aqueles no grupo controle placebo com solução salina normal.

\section{Huang L; Estados} Capdevila, Unidos Analisou a efcácia da aromaterapia na melhoria desempenho do trabalho e na redução do estresse no local de trabalho.

O AG executou a tarefa
do site 2,28 min mais rápido que o $\mathrm{GC}(\mathrm{p}=$ $0,05)$.
L. (2016) precisão os efeitos da estimulação quantitativa do odor nas funções cardiorrespiratórias e a duração do efeito.

\section{O uso de óleos essenciais entre a equipe de enfermagem pode melhorar $\mathrm{o}$ ambiente no local de trabalho e aumentar a satisfação do enfermeiro.}

Os participantes foram divididos

aleatoriamente em um grupo de aromaterapia (AG) e um grupo controle (GC), e foram convidados a participar de uma sessão específica apenas uma vez.
Aromaterapia (inalação de óleo essencial de petitgrain) pode melhorar o desempenho no local de trabalho. 


\section{$\checkmark \quad$ Efetividade do uso da aromaterapia na prática clínica}

O uso aromaterapia na prática possui, conhecidamente, efeitos benéficos sobre diversos cenários clínicos.

Para Lee \& Shin (2017) a aromaterapia utilizando óleo essencial de gengibre promove a redução de náuseas e vômitos após cirurgias abdominais de grande porte. Além de possuir a vantagem de ser um método não invasivo, o óleo de gengibre é barato e de fácil administração, além de não possuir diversos efeitos secundários conhecidos.

Segundo Farahani et al. (2019) a aromaterapia pode ser utilizada como terapia alternativa em pacientes com câncer, melhorando o estado geral destes atuando sobre a ansiedade, especialmente através da inalação e da massagem com lavanda, o que corresponde aos resultados encontrados em outros estudos feitos com pacientes dialíticos, com idosos e com mulheres em trabalho de parto. A massagem com lavanda também se mostrou eficaz contra sintomas de depressão nos pacientes em quimioterapia.

O tratamento da ansiedade e da depressão constitui o principal uso terapêutico da aromaterapia. De acordo com Gnatta, Dornellas, et al.(2011) o óleo de lavanda promoveu a redução do estado de ansiedade na amostra, porém de forma não significativa estatisticamente. Em outro trabalho, Gnatta et al., (2014) verificou que os óleos essenciais de rosa e ylang ylang não alteram de forma significativa a percepção da autoestima. Apesar disso o autor enfatiza que este não é um resultado definitivo e novos trabalhos devem ser realizados para avaliar melhor a a efetividade desses óleos.

Para Lyra et al. (2010) a aromaterapia foi eficaz na diminuição nos níveis de estresse e ansiedade de alunos de graduação na área da saúde, atuando sobre o sistema límbico via bulbo olfatório, sobre a pressão arterial e sobre o sistema nervoso central.

Conforme o estudo feito por Domingos \& Braga (2014) a massagem com aromaterapia em usuários durante internação psiquiátrica trouxe múltiplos benefícios, diminuindo sintomas ansiosos e contribuindo para o tratamento da doença mental.

\section{$\checkmark$ Mecanismo de ação}

Os efeitos terapêuticos dos óleos essenciais ainda não estão totalmente esclarecidos por meio de estudos clínicos científicos, no entanto infere-se que essas moléculas produzam um estímulo capaz de liberar neurotransmissores, como encefalinas e endorfinas, os quais geram um efeito analgésico e produzem uma sensação de bem-estar e relaxamento (Gnatta et al., 2016).

A ação aromaterapêutica de um óleo está relacionada com a via pela qual suas moléculas são administradas. Quando a administração ocorre por via inalatória, as moléculas atravessam o trato respiratório superior, chegando às vias inferiores, onde são absorvidas pelos vasos sanguíneos pulmonares e distribuídas no organismo por meio da corrente sanguínea. Nesta via, as moléculas causam estimulação dos nervos olfativos que, possuem ligação direta com o Sistema Límbico, responsável por emoções, sentimentos, memórias e impulsos (Gnatta, Zotelli, et al., 2011).

As substâncias que constituem os $\mathrm{OE}$ atuam enviando sinais biológicos a células receptoras nas narinas. Após serem recebidos, esses sinais são transmitidos para partes límbicas e hipotálamo do cérebro, através de bulbo olfativo. Como resposta o sistema nervoso central libera neurotransmissores capazes de favorecer o bem-estar psicológico e físico e proporcionar uma sensação de alívio (Ali et al., 2015; Domingos \& Braga, 2014).

Quando o contato se dá através da via cutânea, penetram na pele ou mucosas, devido a suas moléculas pequenas e de baixo peso molecular, são absorvidas e distribuídas pelos tecidos corporais através da corrente sanguínea. Quando ingeridos, as suas moléculas penetram pela mucosa intestinal, alcançam a corrente sanguínea e são distribuídas no organismo (Gnatta et al., 2014, 2016).

Em pesquisas analisadas por Kawai et al. (2017), verificou-se que algumas fragrâncias têm efeitos inibitórios no 
cérebro e nas funções nervosas autonômica. Relataram ainda que a inalação de óleo de pimenta induziu um aumento na concentração plasmática de adrenalina em comparação com o estado de repouso, enquanto a inalação de óleo de rosa causou uma diminuição na concentração de adrenalina e concluíram que a inalação de óleos essenciais pode modular a atividade simpática.

Como exemplo, temos os óleos essenciais de lavanda (Lavandula angustifolia) e de gerânio (Pelargonium graveolens) que apresentam o linalol e o acetato de linalil entre os seus constituentes químicos e possuem ação tranquilizante e calmante. Essa duas substâncias inibem a ação colinérgica através de alterações no funcionamento de canais iônicos nas junções neuromusculares, além disso, promovem inibição do tônus do sistema nervoso central, ao interagirem na ação do neurotransmissor GABA e no sistema dopaminérgico (Domingos \& Braga, 2015).

Alguns estudos comprovam que os óleos essenciais também apresentam efeito antimicrobiano, por conta da sua atuação na estrutura da parede celular do microrganismo, desnaturando e coagulando proteínas. A ação se daria por alterações na permeabilidade da membrana citoplasmática graças ao caráter lipofílico destas moléculas, interrompendo os processos vitais da célula, como transporte de elétrons, translocação de proteínas, fosforilação e outras reações enzima-dependentes, e tais ações resultariam na perda do controle quimiosmótico da célula afetada, destruindo-a (Gnatta et al., 2016).

Dos artigos científicos utilizados neste estudo, apenas 6 deram ênfase ou citaram a fisiologia dos óleos essenciais na prática terapêutica, deste modo fica clara a necessidade do desenvolvimento de novas investigações e estudos com foco na busca de evidências científicas que comprovam os mecanismos de ação da aromaterapia, para oferecer subsídios e novas perspectivas para a área da saúde, do bem-estar e da qualidade de vida.

\section{$\checkmark \quad$ Tipos de Aromaterapia e sua aplicabilidade}

Quanto aos tipos de Aromaterapia e sua aplicabilidade nos artigos estudados, verificou-se que foram feitas pelas vias cutânea e inalatória. Gnatta et al. (2014) utilizaram óleos essenciais de rosa e ylang-ylang pela via inalatória para verificar sua eficácia na melhora da autoestima, não apresentando diferenças significativas.

No trabalho de Domingos \& Braga (2015) foram aplicados os óleos de Lavanda e Gerânio através de massagem por alisamento em pacientes com Transtorno da Personalidade e do Comportamento do Adulto com o objetivo de diminuir a ansiedade dos mesmos. O que foi constatado através dos parâmetros de Frequência Cardíaca e Respiratória. Eficácia também comprovada no estudo de Lyra et al. (2010) em que 36 participantes de uma amostra de 228 alunos do curso da saúde foram submetidos a sessões por inalação com mistura de óleos de Lavanda, Laranja-azeda, ylang-ylang e Cedro para redução da ansiedade e estresse.

Já no estudo de Gnatta, Dornellas, et al. (2011) não encontraram valores expressivos com os óleos de Lavanda e Gerânio no alívio da ansiedade em graduandos de enfermagem.

Johnson et al. (2017) também utilizaram o óleo essencial de lavanda para promover um ambiente terapêutico para enfermeiros em uma unidade de atendimento cirúrgico especializado e unidade de trauma ortopédico através da inalação.

Gnatta et al. (2014) utilizaram o óleo essencial ylang- ylang por meio de aplicação cutânea e inalatória para avaliarem possíveis alterações na percepção da ansiedade e da autoestima e os parâmetros fisiológicos como pressão arterial e temperatura. Apresentou alterações significativas apenas na percepção da ansiedade.

No trabalho de Lee \& Shin (2017) os escores de náusea e vômito apresentaram diminuição significativa no grupo com inalação de óleo essencial de gengibre em pacientes submetidos a cirurgia abdominal.

\section{$\checkmark \quad$ Patologias em que a aromaterapia se configura como prática eficaz}

Acerca das patologias nas quais a aromaterapia se configura como terapêutica eficaz observa-se afecções como o 
estresse no local de trabalho, como experimentado por Huang \& Capdevila (2017) em estudo que analisou a eficácia da aromaterapia na melhoria do desempenho no trabalho e na redução do estresse no local de trabalho.

Outrossim, observou-se a eficiência em aliviar náuseas e vômitos na recuperação de pós operatório, onde Lee \& Shin (2017) demonstraram que os escores de náusea e vômito foram significativamente menores no grupo experimental com inalação de óleo essencial de gengibre do que aqueles no grupo controle placebo com solução salina normal. No grupo experimental, os escores de náusea e vômito diminuíram consideravelmente nas primeiras 6 horas após a aromaterapia inalatória com óleo essencial de gengibre.

Também verificou -se que a aromaterapia reduziu a agitação e os sintomas neuropsiquiátricos em pacientes com demência e mais ainda a intervenção com aromaterapia na internação psiquiátrica em hospitais gerais trouxe melhorias em diversas esferas, como a diminuição dos sintomas ansiosos e no enfretamento do transtorno mental como demonstrado por Domingos e Braga (2014).

\section{$\checkmark$ A aromaterapia como ferramenta para a redução da ansiedade e promoção de bem-estar psicológico e a importância da interprofissionalidade}

As práticas clínicas com Aromaterapia têm se tornado área em expansão para saúde, podendo ser vista como uma das técnicas complementares popularmente utilizadas nas diversas especialidades médicas, tais como a Psiquiatria e Saúde Mental. Existem indicações para o uso da Aromaterapia no tratamento de ansiedade, depressão e outros transtornos psiquiátricos, configurando-se como uma técnica promissora, contudo, essas indicações não são suficientemente comprovadas. Há desse modo, uma escassez de estudos que apresentem a ação da aromaterapia para a população portadora de transtornos psiquiátricos (Domingos \& Braga, 2015).

No âmbito da Psiquiatria e Saúde Mental, os estudos com Aromaterapia investigam a eficácia sobre a redução dos sintomas de quadros demenciais em idosos e sintomas de ansiedade. Desse modo, a Aromaterapia pode representar uma nova ferramenta a ser empregada na melhora dos desequilíbrios físicos ou emocionais de pessoas que sofrem com a ansiedade ou outros transtornos mentais (Gnatta, Dornellas, et al., 2011). A ansiedade é um estado emocional que comporta um conjunto das diferentes experiências humanas, englobando sentimentos de medo, insegurança, apreensão e alteração dos estados de vigília ou alerta. Durante a internação psiquiátrica, expressa-se por meio de componentes psicológicos e físicos, como apreensão, medo, angústia, alterações nos sinais vitais e agitação psicomotora (Batistella et al., 2021).

De modo geral, os benefícios da Aromaterapia na redução dos níveis de estresse podem ser entendidos por meio do relaxamento resultante da exposição ao aroma de óleo essencial. As moléculas químicas volatizadas influenciam a diminuição da atividade do sistema nervoso simpático concomitantemente à estimulação do parassimpático (Montibeler et al., 2018). Além disso, destaca-se a associação de mecanismo molecular em que os óleos essenciais emitem um sinal biológico às células receptoras do nariz, que o transmite ao sistema límbico e hipotálamo, desencadeando a liberação de neurotransmissores (Montibeler et al., 2018). A intervenção com aromaterapia na internação psiquiátrica em hospitais gerais trouxe melhorias em diversas esferas, como a diminuição dos sintomas ansiosos e no enfretamento do transtorno mental, e foram acessadas por meio da percepção que o usuário atribui ao cuidado de enfermagem e ao tratamento (Domingos \& Braga, 2014). Os benefícios da Aromaterapia foram evidentes aos usuários a partir do momento em que experimentaram a diminuição dos sintomas ansiosos, físicos e psíquicos, e na melhoria do padrão de sono.

No estudo-piloto intitulado Efetividade da massagem com Aromaterapia no estresse da equipe de enfermagem do centro cirúrgico, desenvolvido em um centro cirúrgico de um hospital de ensino no interior de São Paulo/Brasil, houve diminuição estatística dos parâmetros biofisiológicos, pressão arterial e frequência cardíaca, mas em se tratando dos aspectos psicológicos, as medidas não evidenciaram efeito sobre o estresse. Neste mesmo estudo, observou-se que a Aromaterapia 
associada à massagem com os óleos essenciais de Lavandula angustifolia e Pelargonium graveolens demonstrou efetividade na redução de parâmetros biofisiológicos dos profissionais da equipe de enfermagem de centro cirúrgico, redução essa evidenciada pela diminuição da frequência cardíaca e pressão arterial. Tais resultados corroboram os encontrados em pesquisas realizadas anteriormente, as quais demonstraram a efetividade da Aromaterapia apenas em parâmetros fisiológicos, suscitando estudos posteriores que possibilitem quantificar as evidências do seu uso em contextos de alta complexidade (Montibeler et al., 2018).

No cenário mundial atual, a Aromaterapia como área em expansão, apresenta-se como ferramenta para o uso na melhora da autoestima, possibilitando às pessoas uma nova chance para que desenvolvam uma visualização positiva de sua autoimagem. $\mathrm{O}$ estudo de campo intitulado $\mathrm{O}$ uso da aromaterapia na melhora da autoestima realizado com os funcionários pertencentes aos setores de Higienização e CME do Hospital Universitário da Universidade de São Paulo (HU-USP), propôs como intervenção o uso da Aromaterapia através da inalação de óleos essenciais de Rosa ou ylang-ylang ou ainda de essência de Rosa (grupo placebo). De acordo com a literatura sobre Aromaterapia, estes dois óleos essenciais são indicados para melhorar a autoestima, embora cada um deles atue de forma sutilmente diferente no sistema límbico (Gnatta, Dornellas, et al., 2011). Nesse estudo, verificou-se que os óleos essenciais de rosa e ylang-ylang não alteraram de forma significativa a percepção da autoestima. Portanto, não foi possível comparar a efetividade entre eles, uma vez que tanto os participantes que utilizaram os óleos essenciais quanto os que usaram a essência de Rosa (placebo) não apresentaram melhora significativa.

A escassez de estudos acerca da Aromaterapia no cuidado ao usuário portador de transtorno mental, amostra pequena e ausência de grupo controle, bem como de cálculo amostral, apresentaram-se como limitações da pesquisa. Diante disso, cabe destacar a necessidade de novos estudos metodologicamente mais elaborados, com amostra populacional significativa, randomizada e controlada (Domingos \& Braga, 2015).

As Práticas Integrativas Complementares (PICs) agrupam abordagens com foco na prevenção de agravos, recuperação da saúde e na promoção do autocuidado. A inserção de grupos como modalidade de atenção coletiva à população é frequente nos serviços de saúde. Busca-se através da organização de grupos otimizar o processo de trabalho e a participação do processo educativo de usuários e de outros profissionais da equipe (Mello et al., 2020), o que correlaciona-se com a prática interprofissional. As ações interprofissionais constituem-se em uma proposta de educação que introduz a reflexão sobre projeto de formação colaborativa e compartilhada para o trabalho, em dimensões interdisciplinares e interprofissionais (Pereira, 2018).

\section{Conclusão}

As Práticas Integrativas e Complementares (PICs), onde a aromaterapia está inserida, contribui sensivelmente com a qualidade de vida da população, buscando a visão holística e integral do indivíduo, e como esta abordagem pode colaborar com a prevenção e tratamento de doenças.

A educação interprofissional em saúde, com as suas competências colaborativas na atenção à saúde, visando o trabalho em equipe, pode lançar mão da Aromaterapia para prestação de orientação e acompanhamento de indivíduos que necessitam ampliar o bem-estar geral. Vale ressaltar que é necessário que os profissionais envolvidos sejam adequadamente capacitados, tanto na aplicação das PICs quanto sobre os fundamentos do trabalho interprofissional

Deste modo acredita-se que este estudo contribuiu no âmbito da prática interprofissional, da formação acadêmica e na área científica, pois através do mesmo pôde se identificar eficácia na utilização de algumas práticas emergentes na atualidade, balizando a necessidade de repensar implementar modelos tanto de ensino como, de extensão e pesquisa, além dos atendimentos. Além disto, estudos futuros podem ser realizados com o objetivo de avaliar abordagens de prática interprofissional em saúde com o uso da Aromaterapia. 


\section{Referências}

Ali, B., Al-Wabel, N. A., Shams, S., Ahamad, A., Khan, S. A., \& Anwar, F. (2015). Essential oils used in aromatherapy: A systemic review. Asian Pacific Journal of Tropical Biomedicine, 5(8), 601-611. https://doi.org/10.1016/j.apjtb.2015.05.007

Andrei, P. \& Del Comune, A. P. (2005). Aromaterapia e suas aplicações. CADERNOS • Centro Universitário S. Camilo, 11(4), 57-68. http://www.saocamilosp.br/pdf/cadernos/36/07_aromaterapia.pdf

Barros, N. F. de Spadacio, C., \& Costa, M. V. da. (2018). Trabalho interprofissional e as Práticas Integrativas e Complementares no contexto da Atenção Primária à Saúde: potenciais e desafios. Saúde Em Debate, 42(1), 163-173. https://doi.org/10.1590/0103-11042018s111

Batistella, C. E., Camilo, I. R., Comparin, K. A., Aragão, F. A., \& Frare, J. C. (2021). Efetividade da terapia floral para redução de sintomas de ansiedade em universitários: ensaio clínico randomizado. Research, Society and Development, 10(1), e44710111926. https://doi.org/10.33448/rsd-v10i1.11926

Brasil. (2018). Portaria $N^{\circ}$ 702, de 21 de março de 2018. Ministério Da Saúde. https://www.in.gov.br/web/guest/materia//asset_publisher/Kujrw0TZC2Mb/content/id/7526450/do1-2018-03-22-portaria-n-702-de-21-de-marco-de-2018-7526446

Domingo, T. D. S., \& Braga, E. M. (2013). Aromaterapia e ansiedade: revisão integrativa da literatura. Cadernos de Naturologia e Terapias Complementares, 2(2), 73. https://doi.org/10.19177/cntc.v2e2201373-81

Domingos, T. da S., \& Braga, E. M. (2014). Significado da massagem com aromaterapia em saúde mental. Acta Paulista de Enfermagem, 27(6), 579-584. https://doi.org/10.1590/1982-0194201400094

Domingos, T. da S., \& Braga, E. M. (2015). Massage with aromatherapy: effectiveness on anxiety of users with personality disorders in psychiatric hospitalization. Revista Da Escola de Enfermagem Da USP, 49(3), 450-456. https://doi.org/10.1590/S0080-623420150000300013

Farahani, M. A., Afsargharehbagh, R., Marandi, F., Moradi, M., Hashemi, S.-M., Moghadam, M. P., \& Balouchi, A. (2019). Effect of aromatherapy on cancer complications: A systematic review. Complementary Therapies in Medicine, 47, 102169. https://doi.org/10.1016/j.ctim.2019.08.003

Gnatta, J. R., Dornellas, E. V., \& Silva, M. J. P. da. (2011). O uso da aromaterapia no alívo da ansiedade. Acta Paulista de Enfermagem, 24(2), 257-263. https://doi.org/10.1590/S0103-21002011000200016

Gnatta, J. R., Kurebayashi, L. F. S., Turrini, R. N. T., \& Silva, M. J. P. da. (2016). Aromatherapy and nursing: historical and theoretical conception. Revista Da Escola de Enfermagem Da USP, 50(1), 127-133. https://doi.org/10.1590/S0080-623420160000100017

Gnatta, J. R., Piason, P. P., Lopes, C. de L. B. C., Rogenski, N. M. B., \& Silva, M. J. P. da. (2014). Aromatherapy with ylang ylang for anxiety and selfesteem: a pilot study. Revista Da Escola de Enfermagem Da USP, 48(3), 492-499. https://doi.org/10.1590/S0080-623420140000300015

Gnatta, J. R., Zotelli, M. F. M., Carmo, D. R. B., Lopes, C. de L. B. C., Rogenski, N. M. B., \& Silva, M. J. P. da. (2011). O uso da aromaterapia na melhora da autoestima. Revista Da Escola de Enfermagem Da USP, 45(5), 1113-1120. https://doi.org/10.1590/S0080-62342011000500012

Huang, L., \& Capdevila, L. (2017). Aromatherapy Improves Work Performance Through Balancing the Autonomic Nervous System. The Journal of Alternative and Complementary Medicine, 23(3), 214-221. https://doi.org/10.1089/acm.2016.0061

Johnson, K., West, T., Diana, S., Todd, J., Haynes, B., Bernhardt, J., \& Johnson, R. (2017). Use of aromatherapy to promote a therapeutic nurse environment. Intensive and Critical Care Nursing, 40, 18-25. https://doi.org/10.1016/j.iccn.2017.01.006

Kawai, E., Nakahara, H., Ueda, S., Manabe, K., \& Miyamoto, T. (2017). A novel approach for evaluating the effects of odor stimulation on dynamic cardiorespiratory functions. PLOS ONE, 12(3), e0172841. https://doi.org/10.1371/journal.pone.0172841

Lee, Y. R., \& Shin, H. S. (2017). Effectiveness of Ginger Essential Oil on Postoperative Nausea and Vomiting in Abdominal Surgery Patients. The Journal of Alternative and Complementary Medicine, 23(3), 196-200. https://doi.org/10.1089/acm.2015.0328

Lyra, C. S. de, Nakai, L. S., \& Marques, A. P. (2010). Eficácia da aromaterapia na redução de níveis de estresse e ansiedade em alunos de graduação da área da saúde: estudo preliminar. Fisioterapia e Pesquisa, 17(1), 13-17. https://doi.org/10.1590/S1809-29502010000100003

Macedo, F. E. B. (n.d.). Práticas Integrativas e Complementares no SUS na produção acadêmica da Educação Física brasileira: uma revisão da literatura. [Universidade Federal do Rio Grande do Sul]. https://lume.ufrgs.br/handle/10183/196551?show=full

Mello, R. C. de, Domingues, N. R. P., Santos, A. K. C. dos, Igeski, T. P. Z., Figueiredo, K. C., \& Silva, D. B. da. (2020). Práticas integrativas e complementares e abordagens grupais na atenção primária. Saúde e Meio Ambiente: Revista Interdisciplinar, 9(Supl.1), 87-88. https://doi.org/10.24302/sma.v9iSupl.1.3395

Montibeler, J., Domingos, T. da S., Braga, E. M., Gnatta, J. R., Kurebayashi, L. F. S., \& Kurebayashi, A. K. (2018). Efetividade da massagem com aromaterapia no estresse da equipe de enfermagem do centro cirúrgico: estudo-piloto. Revista Da Escola de Enfermagem Da USP, 52. https://doi.org/10.1590/s1980-220x2017038303348

Pereira, M. F. (2018). Interprofissionalidade e saúde: conexões e fronteiras em transformação. Interface - Comunicação, Saúde, Educação, 22(2), 1753-1756. https://doi.org/10.1590/1807-57622018.0469 